\title{
OUTCOMES OF SURGICAL RECONSTRUCTION OF THE SUPERIOR VENA CAVA SYNDROME IN PATIENTS WITH LOCALLY ADVANCED THYMOMAS
}

\author{
Valeriy Boyko ${ }^{1}$ \\ Andriy Krasnoyaruzhskyi \\ Department of Thoracoabdominal Surgery ${ }^{l}$ \\ Dmytro Minukhin \\ Department surgery No. $1^{2}$ \\ Dmytro Dubovyk \\ Medical and diagnostic department for adults \\ Medical Center «Pervotsvit» \\ Novooskilska str., 8V, Irpin city, Kyiv region, Ukraine, 08201 \\ Kateryna Ponomarova \\ Department surgery No. $1^{2}$ \\ Anastasiya Sochnieva \\ Department surgery No. $1^{2}$ \\ Vasil Kritsak \\ Department of Thoracoabdominal Surgery ${ }^{l}$

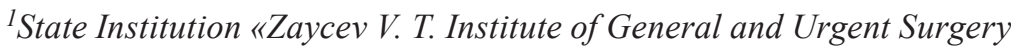 \\ of National Academy of Medical Sciences of Ukraine» \\ 1 Balakireva entry, Kharkiv, Ukraine, 61103 \\ ${ }^{2}$ Kharkiv National Medical University \\ 4 Nauky ave., Kharkiv, Ukraine, 61022
}

\section{Abstract}

The aim of the study: to study and demonstrate the potential and technical aspects of the surgical treatment of locally advanced thymus tumours with the tumour infiltrate affecting superior vena cava and its branches.

Methods. 56 patients with locally advanced thymomas complicated by SVCS were study. The control group included 30 patients with SVCS were treated with conventional techniques, while the experimental group included 26 patients who underwent a novel bypass surgery developed by us.

Results. Based on our observations, patients tolerated these surgeries much better. The venous bypass was mandatorily complemented with cytoreduction. Auriculo-jugular (left and right) and auriculo-subclavian (left and right) bypasses were used in our observations

Complications in the post-operative period were reported from the experimental group and included auriculo-subclavian bypass thrombosis, post-operative complications were reported in the control group including haemorrhage from the sternotomy wound in $1(3.3 \%)$ case, superior vena cava thrombosis in $2(6.6 \%)$ cases, pneumonia in $2(6.6 \%)$ cases and thromboembolism of small pulmonary arteries in $2(6.6 \%)$ cases.

Post-operative lethality in the study groups was reported in the control and in the experimental group. Total lethality rate was $8.9 \%$ (5 patients). The relative risk of complications and lethal outcome was calculated for patients from both groups. It was found that the risk of complications was twice as high in the control group as in the experimental group (standard error of relative risk equals 0.64), whereas the risk of lethal outcome increased by a factor of 3.5 in the control group (standard error of relative risk equals 1.09).

Conclusion. It has been established that the superior vena cava syndrome in patients with locally advanced thymoma is an emergency condition whose surgical correction must be personalised depending on the anatomic and topographic classification of SVC lesion types. It is known that an obligatory pre-condition of the perioperative period in this category of patients is an adequate 
vascular approach to the superior vena cava system. The first mandatory step of the radical surgery in patients with locally advanced thymomas with SVC invasion should be the auriculo-jugular and auriculo-subclavian bypasses, which can reduce the relative risk of post-operative complications by a factor of the risk of lethal by a factor of 3.5.

Keywords: superior vena cava syndrome, thymoma, surgical treatment, postoperation complication, radical surgery, bypass.

DOI: $10.21303 / 2504-5679.2021 .001852$

\section{Introduction}

The superior vena cava syndrome (SVCS) was first described in 1757 [1, 2]. Reduced blood flow in the superior vena cava (SVC) system decreases the amount of venous blood in the right auricle, causing a number of clinical characteristics and symptoms, such as dilated neck veins, oedematous upper limbs, cyanosis, headache, dyspnoea, cough, and dysphagia [3,4]. Impaired blood flow may be caused by external compression, direct tumour invasion, thrombosis, or ineffective venous return as a consequence of diseases affecting the right auricle or the vessel lumen itself. Over time, the main role in the aetiology of this syndrome shifted from tuberculosis and syphilitic aneurisms, prevalent in the beginning of the $20^{\text {th }}$ century, to locally advanced mediastinum tumours, such as thymomas [5]. In the USA, 15,000 new SVCS cases are reported annually, up to $85 \%$ of which are developed due to a primary or secondary tumour lesion. Around $73-85 \%$ of all CVCS cases of tumour genesis are due to locally advanced thymomas $[6,7]$.

The first reliable description of the clinical profile of SVCS was provided by W. Hunter $(1757)[8,9]$. Further numerous works of foreign and Ukrainian authors considerably expanded the understanding of the origin of this syndrome; however, surgical treatment methods were not used until the middle or end of the previous century. The first mentions of successful azygo-atrial anastomosis for SVCS date back to $1951[10,11]$. In the same year, Sampsen combined the azygos vein with the right atrium using venous autotransplant. A successful application of a synthetic transplant for the plastic replacement of superior vena cava was first reported by Blondean et al. in 1959 [12, 13].

SVCS is also an emergency condition related to impaired blood flow in the SVC system and being one of the variants of compression mediastinum syndrome [14, 15]. The disease is clinically manifested as SVCS, uni- or bilateral Paget-Schroetter syndrome. Progressive venous oedema of the upper half of the body and upper limbs significantly deteriorates brain haemodynamics, which can lead to brain oedema and herniation $[16,17]$. Taking into account the fact that, with the development of circulatory hypoxia, the blood flow to the right heart compartments, the circulating blood volume (CBV) and the cardiac output are reduced, this type of tumour can be considered a life-threatening condition $[18,19]$. Previously, the tactics in this clinical situation included chemo- and radiation therapy. Experience shows that these conservative measures require much time, while thymomas may be resistant to chemo- and radiation therapy. We have recently adopted active personalised surgical tactics, which largely depends on the interaction between the tumour, SVC and its branches [20].

The aim of the study is to demonstrate the potential and technical aspects of the surgical treatment of locally advanced thymus tumours with the tumour infiltrate affecting superior vena cava and its branches.

\section{Materials and research methods}

The study is a retrospective analysis of observation data involving 56 patients with locally advanced thymomas complicated by SVCS who were treated at the Department of Thoracoabdominal Surgery of the State Institution «Zaycev V. T. Institute of General and Urgent Surgery of the National Academy of Medical Sciences of Ukraine» between 2014 and 2020. The control group included 30 patients with SVCS treated with conventional techniques, while the experimental group included 26 patients who underwent a novel bypass surgery developed by us.

The authors declare that all procedures and experiments of this study respect the ethical standards in the Helsinki Declaration of 1975, as revised in 2008 (5), as well as the national law. Informed consent to participate in the study was discussed and signed by all study participants. Meeting of the Bioethics Commission (protocol No. 2 11.03.2021) of the State Institution «Zaycev V. T. Institute of General and Urgent Surgery of the National Academy of Medical Sciences of Ukraine». 
At the preoperative stage, all patients underwent standard diagnostic tests to assess the advancement of the tumour process in the mediastinum and functional reserves: multislice computed tomography (MSCT) of thoracic and abdominal organs, comprehensive ultrasound test (including mediastinum ultrasound), electrocardiography, echocardiography, spirometry. Fiber-optic bronchoscopy was performed in case of lung lesion. The most reliable method of diagnosing SVC invasion at the pre-operative stage and assessing tumour advancement is considered to be MSCT with intravenous contrast enhancement with iso-osmolar contrast, which does not cause a sharp blood pressure drop that is likely to cause a vascular collapse at the backdrop of reduced heart flow. $\mathrm{SVC}$ invasion is most precisely visualized in the early venous phase. Echocardiography is required to identify a tumour thrombus in the mediastinum. Besides, patients obligatorily underwent doppler ultrasound test of upper limb and neck vessels.

There was not found a significant difference in the number of patients, average age, gender composition, or body mass index (BMI) found between OA groups $(p>0.05)$.

The femoral vein was catheterized to ensure continuous venous access, and the subclavian and the jugular veins were catheterized to monitor the central venous pressure.

Taking into account the anatomical and topographic features of superior vena cava and inflowing veins, we have developed a classification of SVC lesion types (working classification of SI «Zaycev V. T. Institute of General and Urgent Surgery of the NAMS of Ukraine») (Fig. 1).

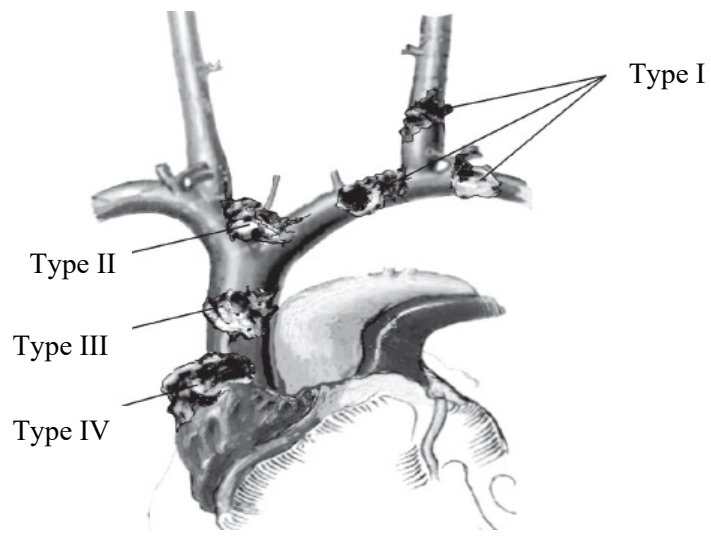

Fig. 1. Types of lesions of superior vena cava and its branches

According to the classification, 4 types of SVC lesions were singled out. Type I stands for lesions in the left brachiocephalic vein, subclavian and jugular veins (uni- or bilateral); Type II stands for lesions in the upper SVC segment; Type III stands for lesions in the middle SVC segment; Type IV stands for lesions in the lower SVC segment. Table 1 presents the distribution of patients in the experimental and control groups depending on the SVC lesion type. The difference between the groups by lesion types is not statistically significant (the chi-squared value equals 0.48 at $\mathrm{p}$-value $=0.92$ ). There was not found a significant difference in the number of patients, average age, gender composition, or body mass index (BMI) found between OA groups $(p>0.05)$.

Table 1

Structure of study groups according to the SVC lesion type

\begin{tabular}{ccccc}
\hline \multirow{2}{*}{ SVC lesion type } & \multicolumn{2}{c}{ Experimental group $\boldsymbol{n = 2 6}$} & \multicolumn{2}{c}{ Control group $\boldsymbol{n = 3 0}$} \\
\cline { 2 - 5 } & $\boldsymbol{n}$ & $\mathbf{\%}$ & $\boldsymbol{n}$ & $(30 \%)$ \\
\hline Type 1 & 10 & $(38.5 \%)$ & 9 & $(33.3 \%)$ \\
Type II & 8 & $(30.8)$ & 10 & $(26.7 \%)$ \\
Type III & 6 & $(23 \%)$ & 8 & $(10 \%)$ \\
Type IV & 2 & $(7.7 \%)$ & 3 & $(100 \%)$
\end{tabular}


The studied group are equivalent and comparable in terms of basic prognosis criteria, sex, age, stage of tumor and localization, as well as by the degree of severity of clinical signs.

Patients in the study groups also had concomitant diseases that significantly influenced the course of the main disease, patients' preparation for the surgery and characteristics of the peri- and postoperative period. All concomitant diseases were identified at the pre-hospital stage thanks to thorough examination. Cardiovascular diseases were the most common in $22(39.3 \%),(p<0.05)$ patients (hypertension, ischemic heart disease, various arrhythmias) followed by respiratory diseases in $9(16.1 \%),(p<0.05)$ patients (pulmonary emphysema, chronic obstructive pulmonary disease, bronchial asthma); myasthenia in $14(25 \%),(p<0.05)$ patients, diabetes mellitus in $7(12.5 \%),(p<0.05)$ patients, and thyroid gland diseases in $5(8.9 \%),(p<0,05)$ patients (multinodular euthyroid goiter, autoimmune thyroiditis). The structure of concomitant pathologies is provided in Table 2. It should be noted that one patient can have several concomitant pathologies, but the table includes only the most pronounced ones. The difference between groups by concomitant pathology types is not statistically significant (the chisquared value equals 2.14 at $p$-value $=0.71$ ). The differences between the main and control groups are statistically significant $(p<0.05)$ (Table 1).

Table 2

Structure of concomitant pathologies in study groups

\begin{tabular}{ccc}
\hline Concomitant diseases & Experimental group $\boldsymbol{n}=\mathbf{2 6}$ & Control group $\boldsymbol{n}=\mathbf{3 0}$ \\
\hline Cardiovascular diseases & $12(46.2 \pm 0.5 \%)$ & $10(33.4 \pm 0.3 \%)$ \\
Respiratory diseases & $4(15.3 \pm 0.1 \%)$ & $4(13.3 \pm 0.2 \%)$ \\
Myasthenia & $6(23.1 \pm 0.3 \%)$ & $8(26.7 \pm 0.1 \%)$ \\
Diabetes mellitus & $3(11.5 \pm 0.3 \%)$ & $4(13.3 \pm 0.01 \%)$ \\
Thyroid gland diseases & $1(3.9 \pm 0.01 \%)$ & $4(13.3 \pm 0.01 \%)$
\end{tabular}

The differences between the main and control groups are statistically significant $(p<0.05)$ (Table 2).

In order to assess the operative risk of complications in patients from the study groups, the ASA physical status classification system was used. We used the scale from the 2014 revision. The distribution of patients depending on the risk score is presented in Table 3. The difference between groups by the perioperative risk score is not statistically significant (the chi-squared value equals 0.04 at $\mathrm{p}$-value $=0.98$ ).

Table 3

Distribution of patients in the study group by the perioperative risk score (ASA)

\begin{tabular}{cccc}
\hline \multirow{2}{*}{ Perioperative risk score } & \multicolumn{3}{c}{ Patient group } \\
\cline { 2 - 4 } & Experimental group $(\boldsymbol{n = 2 6 )}$ & Control group $(\boldsymbol{n}=\mathbf{3 0})$ & Total $(\boldsymbol{n}=\mathbf{5 6})$ \\
\hline ASA II & $17(65.4 \pm 0.2 \%)$ & $20(66.7 \pm 0.1 \%)$ & $37(66.1 \pm 0.3 \%)$ \\
ASA III & $6(23.1 \pm 0.1 \%)$ & $7(23.3 \pm 0.2 \%)$ & $13(23.2 \pm 0.2 \%)$ \\
ASA IV & $3(11.5 \pm 0.3 \%)$ & $3(10.0 \pm 0.3 \%)$ & $6(10.7 \pm 0.01 \%)$
\end{tabular}

Taking into account data from Table 3, it can be observed that most of the patients under study had ASA grade II and III of the perioperative risk. There were no significant differences between study groups in terms of lesion types, concomitant pathologies, and perioperative risk score $(p<0.05)$. The differences between the main and control groups are statistically significant $(p<0.05)$ (Table 3).

Patients from study groups underwent surgeries after thorough examination and assessment of the operative risk. The structure of surgical interventions is described in Table 4. 
Table 4

Structure of surgical interventions in study groups

\begin{tabular}{ccc}
\hline Tactics & Experimental group $(\boldsymbol{n}=\mathbf{2 6})$ & Control group \\
\hline Explorative interventions with biopsy & - & $6(20 \pm 0,2 \%)$ \\
Decompression cytoreductive interventions & - & $4(3.3 \pm 0,1 \%)$ \\
Bypass surgeries & $12(46.2 \pm 0.5 \%)$ & - \\
Extended thymectomy with brachiocephalic vein resection & $9(34.6 \pm 0,1 \%)$ & $13(43.3 \pm 0.3 \%)$ \\
Extended thymectomy with superior vena cava resection & $5(19.2 \pm 0.3 \%)$ & $7(23.4 \pm 0.2 \%)$
\end{tabular}

Ethics review. All patients signed a voluntary informed consent for the examination and treatment, as well as publication of anonymized personal medical information for academic purposes.

Statistical analysis. Findings concerning the effectiveness of different surgical interventions were analysed using non-parametric statistical techniques (analysis of two-dimensional frequency distributions based on the chi-squared criterion and relative risk) in R and RStudio free-access software for statistical calculations and MS Excel spreadsheet processor. The differences between the main and control groups are statistically significant $(p<0.05)$ (Table 4).

\section{Results}

Ensuring adequate vascular approach to inject medicines, carry out infusion therapy and perform anaesthesia is crucial in the perioperative period of patients with locally advanced thymomas with SVCS. Traditional infusion in the SVC system veins significantly increases venous hypertension and oedema of the upper half of the body. To avoid this, femoral veins were catheterized and the medicines were administered intra-arterially (mostly in the radial artery). In patients from the experimental group, where the tumour could not be resected for technical reasons, venous bypass was performed to correct SVCS. Based on our observations, patients tolerated these surgeries much better. The venous bypass was mandatorily complemented with cytoreduction. Auriculojugular (left and right) and auriculo-subclavian (left and right) bypasses were used in our observations (Fig. 2). Since invasive thymomas are most commonly located on the left side [2], left-side bypasses were used more often in SVCS correction.

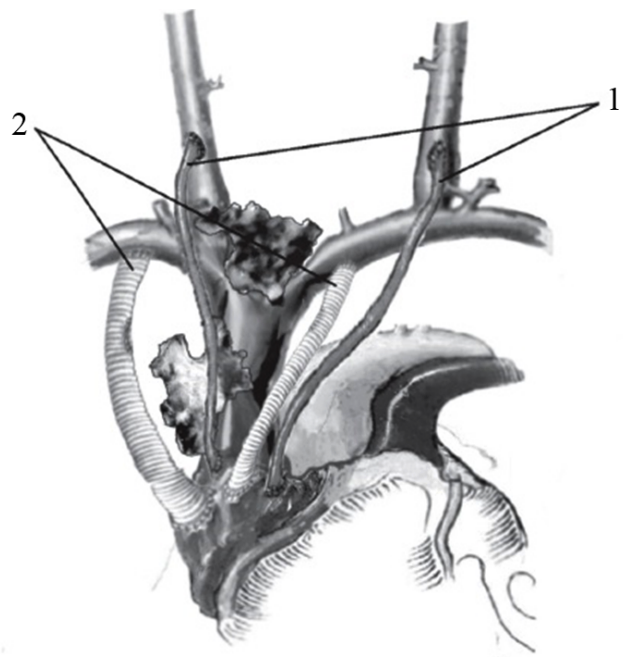

Fig. 2. Auriculo-jugular (1) and auriculo-subclavian (2) bypasses

In $5(19.2 \%)$ cases, the auriculo-jugular bypass was used as an auxiliary stage in radical resection of locally advanced thymoma tumours. In our opinion, resecting a tumour with SVC invasion without a prior bypass is a mistake that may cause a lethal outcome. SVC transection or its acute thrombosis during the tumour resection causes a rapid increase in the central venous pressure 
with all the ensuing consequences. In certain cases, the near-tumoral thrombus or tumoral tissue may get detached and migrate to the right heart compartments, leading to the development of the pulmonary embolism. Therefore, type I, II and III tumour invasions in the SVC were considered clinical situations, for which the auriculo-jugular bypass was mandatory as the first stage of a radical surgery. An important pre-condition of a SVC surgery is the observation of measures aimed to prevent embolic complications. To introduce a prosthesis to the blood flow, first the forceps from the proximal bypass segment are removed, then, after the prosthesis is filled with blood and free from air, the distal forceps located closer to the auricle are removed. While exploring the technique of resecting locally advanced thymomas with SVC invasion, we used the full vertical sternotomy approach extended to the neck along the sternocleidomastoid muscle.

Later, such traumatic approach could be avoided: the proximal anastomosis was formed from a mini-approach on the neck, and the distal one from the sternotomy approach. In the case of large tumours, their resection was only possible after a pericardium incision and specification of the tumour invasion degree from the pericardial cavity. Depending on the degree of pericardium lesion, limited pericardiectomy was performed in the invasion area with the resection of $1 / 3,2 / 3$ and $3 / 4$ of affected pericardium; in $2(7.7 \%)$ patients, full pericardiectomy was performed. In all cases of extended thymectomies, we aimed to preserve diaphragmatic nerves. In case of tumour invasion in the azygos vein, it was preliminarily secured. In Type IV tumour invasion in SVC, it is crucial to preserve the auricular SVC segment that includes the synoauricular node. If this segment cannot be preserved for technical reasons and must be resected, epicardial electrocardiostimulation is indicated, which can be substituted with endocardial stimulation if necessary.

Taking into account that all patients belonged to stage III-IV of the tumour process extent according to Masaoka-Koga's classification, during the post-operative period the patients underwent external-beam radiation therapy according to the split-extended scheme with fraction radiation with single boost dose $(\mathrm{SBD})=2$ Gy once a week up to total boost dose (TBD) $=40-45$ Gy (in radical interventions) and $\mathrm{SBD}=2$ Gy 5 times a week up to TBD 60-70 Gy. Chemotherapy was used in combination with radiation therapy in $11(19.7 \%)$ patients after cytoreductive and explorative interventions.

Complications in the post-operative period were reported in $3(11.5 \%)$ patients from the experimental group and included auriculo-subclavian bypass thrombosis in 1 (3.8\%) case, pneumonia in $1(3.8 \%)$ case and thromboembolism of small pulmonary arteries in $1(3.8 \%)$ case. $7(23.4 \%)$ post-operative complications were reported in the control group including haemorrhage from the sternotomy wound in $1(3.3 \%)$ case, superior vena cava thrombosis in $2(6.6 \%)$ cases, pneumonia in $2(6.6 \%)$ cases and thromboembolism of small pulmonary arteries in $2(6.6 \%)$ cases.

Post-operative lethality in the study groups was reported for $4(13.3 \%)$ patients in the control group and $1(3.8 \%)$ patient in the experimental group. Total lethality rate was $8.9 \%$ (5 patients). The causes of post-operative lethality included myasthenic crisis in 1 (1.8\%) patient, pneumonia in $1(1.8 \%)$ patient, myocardial infarction in $1(1.8 \%)$ patient, and pulmonary embolism in 2 (3.6\% patients).

The relative risk of complications and lethal outcome was calculated for patients from both groups. It was found that the risk of complications was twice as high in the control group as in the experimental group (standard error of relative risk equals 0.64 ), whereas the risk of lethal outcome increased by a factor of 3.5 in the control group (standard error of relative risk equals 1.09).

\section{Discussion}

In our research, which is a retrospective analysis of observation of the results of surgical treatment for 56 patients with locally advanced thymomas complicated by SVCS. The control group included 30 patients with SVCS treated with conventional techniques, while the experimental group included 26 patients who underwent a new bypass surgery developed by us.

In patients with locally advanced thymomas, constantly increased pressure in the SVC system for several months leads to progressive dilatation and development of a network of collateral venous blood flow. Owing to this mechanism, the SVCS remains at the compensation or subcompensation stage for a rather long time [15]. 
It should be noted that in the structure of complications in the post-operative period in our study, the leading place is occupied by auriculo-subclavian bypass thrombosis in $3.8 \%$ of patients. So, in our study, pneumonia was in $3.8 \%$ of patients. Thromboembolism of small pulmonary arteries in $3.8 \%$ of patients whereas in $23.4 \%$ of patients post-operative complications were reported in the control group including haemorrhage from the sternotomy wound in $3.3 \%$ of patients. Also, superior vena cava thrombosis in $6.6 \%$ of patients while pneumonia in $6.6 \%$ of patients. However, thromboembolism of small pulmonary arteries in $6.6 \%$ of patients $[3,10]$.

Study limitations. This study combines the results of a retrospective analysis of observation of the results of surgical treatment patients with locally advanced thymomas complicated by SVCS. Since the use of the venous bypass to improve results in the postoperative period and realize the full effect of treatment in patients.

Prospects for the future research. Since the usage of by-pass methods of surgical treatment and conservative treatment patients with locally advanced thymomas complicated by SVCS can notice to improving of surgical results, we plan to develop an algorithm that will reduce the number of postoperative complications.

\section{Conclusions}

The superior vena cava syndrome in 56 patients $(26$ (46\%) in the experimental group and 30 (54\%) in the control group) with locally advanced thymoma is an emergency condition whose surgical correction must be personalised depending on the anatomic and topographic classification of SVC lesion types.

An obligatory pre-condition of the perioperative period in this category of patients is an adequate vascular approach to the superior vena cava system.

The first mandatory step of the radical surgery in patients with locally advanced thymomas with SVC invasion should be the auriculo-jugular ASA II $37(66.1 \%),(p<0.05)$ and auriculosubclavian bypasses in $13(23.2 \%),(p<0.05)$ which can reduce the relative risk of post-operative complications by a factor of 2 and the risk of lethal by a factor of 3.5 .

\section{Conflict of interests}

The authors declare that they have no conflicts of interest.

\section{Acknowledgment}

The topic of GDR surgery of the thoracic cavity of the State Institution «Zaycev V. T. Institute of General and Urgent Surgery of the National Academy of Medical Sciences of Ukraine», P.01.14, Development of surgical tactics with thymomas complicated by the superior vena cava syndrome, No. UA 0119U002466. This research became a fragment of this research work.

\section{References}

[1] Khaki, F., Javanbakht, J., Sasani, F., Gharagozlou, M. J., Bahrami, A., Moslemzadeh, H., Sheikhzadeh, R. (2013). Cervical type AB thymoma (Mixed) tumour diagnosis in a mynah as a model to study human: clinicohistological, immunohistochemical and cytohistopathological study. Diagnostic Pathology, 8 (1). doi: http://doi.org/10.1186/1746-1596-8-98

[2] Giannopoulou, A., Gkiozos, I., Harrington, K. J., Syrigos, K. N. (2013). Thymoma and radiation therapy: a systematic review of medical treatment. Expert Review of Anticancer Therapy, 13 (6), 759-766. doi: http://doi.org/10.1586/era.13.54

[3] Rouquette, I., Taranchon-Clermont, E., Gilhodes, J., Bluthgen, M.-V., Perallon, R., Chalabreysse, L. et. al. (2019). Immune biomarkers in thymic epithelial tumors: expression patterns, prognostic value and comparison of diagnostic tests for PD-L1. Biomarker Research, 7 (1). doi: http://doi.org/10.1186/s40364-019-0177-8

[4] Wheler, J., Hong, D., Swisher, S. G., Falchook, G., Tsimberidou, A. M., Helgason, T. et. al. (2013). Thymoma Patients Treated in a Phase I Clinic at MD Anderson Cancer Center: Responses to mTOR Inhibitors and Molecular Analyses. Oncotarget, 4 (6), 890-898. doi: http://doi.org/10.18632/oncotarget.1015

[5] Michels, G., Drebber, U., Pfister, R. (2012). Thymoma - an important differential diagnosis of mediastinal tumours. Acta Clinica Belgium, 67 (4), 304-305.

[6] Girard, N., Ruffini, E., Marx, A., Faivre-Finn, C., Peters, S. (2015). Thymic epithelial tumours: ESMO Clinical Practice Guidelines for diagnosis, treatment and follow-up. Annals of Oncology, 26, v40-v55. doi: http://doi.org/10.1093/annonc/mdv277 
[7] Klimova, E. M., Drozdova, L. A., Lavinskaya, E. V., Minukhin, D. V., Kudrevich, A. N. (2020). Advisability of thymectomy in young patients with thymus-independent myasthenia gravis in the presence of biomarkers specific for patients with thymoma. Kharkiv Surgical School, 3, 62-67. doi: http://doi.org/10.37699/2308-7005.3.2020.13

[8] Boyko, V. V., Klimova, O. M., Lavinskaya, O. V., Drozdova, L. A., Minukhin, D. V., Yevtushenko, D. O. (2019). The Choice of Treatment in Various Ages Patients with Myasthenia Againstto Immuneresistance Parameters. International Journal of Education and Science, 2 (3). doi: http://doi.org/10.26697/ijes.2019.3.4

[9] Toker, A., Sonett, J., Zielinski, M., Rea, F., Tomulescu, V., Detterbeck, F. C. (2011). Standard Terms, Definitions, and Policies for Minimally Invasive Resection of Thymoma. Journal of Thoracic Oncology, 6 (7), S1739-S1742. doi: http://doi.org/10.1097/ jto.0b013e31821ea553

[10] Yang, Y., Dong, J., Huang, Y. (2016). Thoracoscopic thymectomy versus open thymectomy for the treatment of thymoma: A meta-analysis. European Journal of Surgical Oncology (EJSO), 42 (11), 1720-1728. doi: http://doi.org/10.1016/j.ejso.2016.03.029

[11] Barbolina, T. D., Bychkov, M. B., Allakhverdiev, A. K., Borisova, T. N., Vladimirova, L. Yu., Gerasimov, S. S. et. al. (2021). Prakticheskie rekomendatsii po lekarstvennomu lecheniyu opukholey vilochkovoy zhelezy (timomy i raka timusa). Malignant Tumours, 10 (3s2-1), 603-614. doi: http://doi.org/10.18027/2224-5057-2020-10-3s2-35

[12] Maury, J.-M., Merveilleux du Vignaux, C., Drevet, G., Zarza, V., Chalabreysse, L., Maisse, C. et. al. (2019). Activation of the mTOR/ Akt pathway in thymic epithelial cells derived from thymomas. PLOS ONE, 14 (3), e0197655. doi: http://doi.org/ 10.1371/journal.pone.0197655

[13] Pikin, O. V., Trakhtenberg, A. Kh., Kolbanov, K. I., Glushko, V. A., Kazakevich, V. I, Amiraliev, A. M. et. al. (2013). Circumferential inferior vena cava resection without prosthesis in patients with mediastinal tumor complicated by mediastinal compression syndrome. Oncosurgery, 2, 60-66.

[14] Alexandrov, O. A., Ryabov, A. B., Pikin, O. V. (2017). Thymoma (review of the literature). Siberian Journal of Oncology, 16 (4), 76-83. doi: http://doi.org/10.21294/1814-4861-2017-16-4-76-83

[15] Lee, G. D., Kim, H. R., Choi, S. H., Kim, Y. H., Kim, D. K., Park, S. I. (2016). Prosthetic graft interposition of the brachiocephalic veins or superior vena cava combined with resection of malignant tumours: graft patency and risk factors for graft occlusion. Journal of Thoracic Disease, 8 (1), 61-7. doi: http//doi.org/10.3978/j.issn.2072-1439.2016.01.07

[16] Okereke, I. C., Kesler, K. A., Rieger, K. M., Birdas, T. J., Mi, D., Turrentine, M. W., Brown, J. W. (2010). Results of Superior Vena Cava Reconstruction With Externally Stented-Polytetrafluoroethylene Vascular Prostheses. The Annals of Thoracic Surgery, 90 (2), 383-387. doi: http://doi.org/10.1016/j.athoracsur.2010.04.004

[17] Ruffini, E., Guerrera, F., Filosso, P. L., Bora, G., Nex, G., Gusmano, S. et. al. (2014). Extended transcervical thymectomy with partial upper sternotomy: results in non-thymomatous patients with myasthenia gravis. European Journal of Cardio-Thoracic Surgery, 48 (3), 448-454. doi: http://doi.org/10.1093/ejcts/ezu442

[18] Odaka, M., Tsukamoto, Y., Shibasaki, T., Mori, S., Asano, H., Yamashita, M., Morikawa, T. (2017). Surgical and oncological outcomes of thoracoscopic thymectomy for thymoma. Journal of Visualized Surgery, 3, 54. doi: http://doi.org/10.21037/ jovs.2017.03.18

[19] Venuta, F., Rendina, E. A. (2012). Superior vena cava resection and reconstruction. European Journal of Cardio-Thoracic Surgery, 41 (5), 1177-1178. doi: http://doi.org/10.1093/ejcts/ezr266

[20] Mayhew, D., Mendonca, V., Murthy, B. V. S. (2019). A review of ASA physical status - historical perspectives and modern developments. Anaesthesia, 74 (3), 373-379. doi: http://doi.org/10.1111/anae.14569

How to cite: Boyko, V., Krasnoyaruzhskyi, A., Minukhin, D., Dubovyk, D., Ponomarova, K., Sochnieva, A., Kritsak, V. (2021). Outcomes of surgical reconstruction of the superior vena cava syndrome in patients with locally advanced thymomas. EUREKA: Health Sciences, 3, 37-44. doi: http://doi.org/10.21303/2504-5679.2021.001852 